\title{
Flat band and Planckian metal
}

\author{
G.E. Volovik ${ }^{1,2}$ \\ ${ }^{1}$ Low Temperature Laboratory, Aalto University, P.O. Box 15100, FI-00076 Aalto, Finland \\ ${ }^{2}$ Landau Institute for Theoretical Physics, acad. Semyonov av., 1a, 142432, Chernogolovka, Russia
}

(Dated: July 29, 2019)

\begin{abstract}
We discuss the recent extension of the Sachdev-Ye-Kitaev (SYK) microscopic mode1 1 , which demonstrates the characteristic features of the Khodel-Shaginyan fermion condensate ${ }^{2}$ - the existence of the finite region of momenta, where the energy of electrons is exactly zero (the flat band). The microscopic derivation of the flat band in this interacting model supports the original idea of Khodel and Shaginyan based on the phenomenological approach. It also suggests that it is the flat band, which is responsible for the linear dependence of resistivity on temperature in "strange metals".
\end{abstract}

PACS numbers:

Recently, in the paper "Theory of the Planck metal", Patel and Sachdev considered a model of interacting fermions which describes a Planckian metal at low temperatures, in order to explain the linear temperature dependence of their resistivity ${ }^{1}$ We show here that the proposed scenario actually describes the formation of the Khodel-Shaginyan fermion condensate ${ }^{2}$ (the flat band). This supports the idea that the flat band is responsible for the linear dependence of resistivity on temperature in "strange metals".

There are different potential sources of the formation of the electronic flat band with zero energy, see e.g. Ref ${ }^{3}$. In particular it can be formed due to electron-electron interaction. The flat band formed by interaction has been first discussed by Khodel and Shaginyan (KS) in $1990^{2}$, who used the phenomenological Landau theory of Fermi liquid, see alss ${ }^{4} 7$ and Fig. 1. This dispersionless energy spectrum has a singular density of states. As a result the superconducting gap and transition temperature are proportional to the coupling constant instead of the exponential suppression in conventional metals with Fermi surfaces. For nuclear systems the linear dependence of the superconducting gap on the coupling constant has been found by Belyae $\sqrt{8}$. In a more rigourous manner the flat band induced by interaction has been obtained in Refs. 910 . Experimentally the merging of levels at the Fermi surface due to interaction has been reported in Refs. 11112

In twisted bilayer graphene there is indication that interaction leads to the further flattening of the spectrum $\frac{13[14}{1 n}$ addition to the geometrical/topological flattening caused by the magic angle twist $\frac{15 / 16}{\text {. }}$

In recent paper by Patel and Sachdev 1 the lattice extension of the Sachdev-Ye-Kitaev (SYK) model has been used to study the problem of the "bad metal" with the universal linear dependence of resistivity on temperature 17 20. However, it appears that signatures of the KS flat band in Figure 1 (right panel) are very similar to those in Figs. 2a and 3a from Patel and Sachdev (PS) ${ }^{11}$. Indeed, Fig. 2a from the PS paper shows the occupancy $n(\mathbf{p})$, which exhibits the same behavior as $n(\mathbf{p})$ in Fig. 1 (right panel), with the finite region where $0<n(\mathbf{p})<1$.

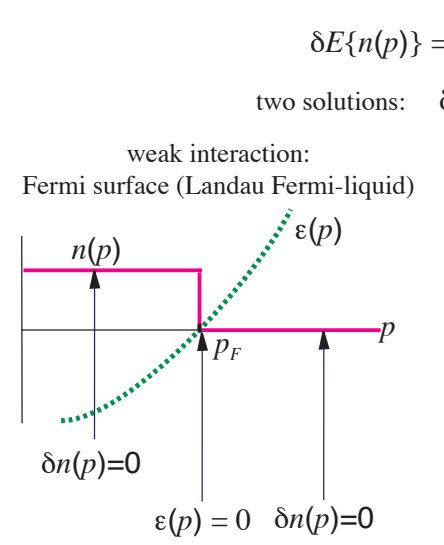

FIG. 1: Consequences of Landau theory of Fermi liquid. Variation of the energy functional over the occupancy $n(\mathbf{p})$ gives two possible solutions: $\epsilon(\mathbf{p})=0$ and $\delta n(\mathbf{p})=0(n(\mathbf{p})=0$ or $n(\mathbf{p})=1)$. left: The Landau Fermi liquid, where the solution $\epsilon(\mathbf{p})=0$ takes place on Fermi surface. Outside of Fermi surface one has either $n(\mathbf{p})=0$ or $n(\mathbf{p})=1$. right: Khodel-Shaginyan flat band, where the solution $\epsilon(\mathbf{p})=0$ takes place in the finite region of momentum space. In this region $0<n(\mathbf{p})<1$.

According to Khodel-Shaginyan, in this region the quasiparticle energy should be zero. And this is clearly seen from the electron spectral density shown in Fig. 3a of the PS paper. So one may conclude that the extended SYK model provides another possible realization of the KS flat band.

That is why the extended SYK model can be used for studying different properties of the materials which experience formation of the KS flat band, including possibly the "bad metal" behavior. In this model, the universal linear dependence of resistivity on temperature has been obtained $d^{11}$ in the regime, where the signatures of the flat band are transparent. From that one may conclude that the phenomenon of Planck metal or bad metal is the consequence of the Khodel-Shaginyan flat band emerging in this model. The idea that the flat band may serve as the origin of the "strange metal" behavior has been suggested 
earlier, see e.g. Ref.21, and recent papers 2223 .

This work has been supported by the European Research Council (ERC) under the European Union's Hori- zon 2020 research and innovation programme (Grant Agreement No. 694248).
1 A.A. Patel and S. Sachdev, Theory of a Planckian metal, arXiv:1906.03265

2 V.A. Khodel and V.R. Shaginyan, Superfluidity in system with fermion condensate, JETP Lett. 51, 553 (1990).

3 G.E. Volovik, Graphite, graphene and the flat band superconductivity, Pis'ma ZhETF 107, 537-538 (2018), JETP Lett. 107, 516-517 (2018),

4 G.E. Volovik, A new class of normal Fermi liquids, JETP Lett. 53, 222-225 (1991).

${ }^{5}$ P. Nozieres, Properties of Fermi liquids with a finite range interaction, J. Phys. (Fr.) 2, 443 (1992).

${ }^{6}$ G.E. Volovik, On Fermi condensate: near the saddle point and within the vortex core, JETP Lett. 59, 830 (1994).

7 T.T. Heikkilä and G.E. Volovik, Flat bands as a route to high-temperature superconductivity in graphite, in: $B a$ sic Physics of Functionalized Graphite, Springer 2016, pp. 123-143.

8 S.T. Belyaev, On the nature of the first excited states of even-even spherical nuclei, JETP 12, 968-976 (1961).

9 D. Yudin, D. Hirschmeier, H. Hafermann, O. Eriksson, A.I. Lichtenstein, M.I. Katsnelson, Fermi condensation near van Hove singularities within the Hubbard model on the triangular lattice, Phys. Rev. Lett. 112, 070403 (2014).

10 S.-S. Lee Non-Fermi liquid from a charged black hole: A critical Fermi ball, Phys. Rev. D 79, 086006 (2009).

11 A.A. Shashkin, V.T. Dolgopolov, J.W. Clark, V.R. Shaginyan, M.V. Zverev and V.A. Khodel, Merging of Landau levels in a strongly-interacting two-dimensional electron system in silicon, Phys. Rev. Lett. 112, 186402 (2014).

12 M.Yu. Melnikov, A.A. Shashkin, V.T. Dolgopolov, S.-H. Huang, C.W. Liu, S.V. Kravchenko, Indication of band flattening at the Fermi level in a strongly correlated electron system, Scientific Reports 7, 14539 (2017).

13 D. Marchenko, D. V. Evtushinsky, E. Golias, A. Varykhalov, Th. Seyller, O. Rader, Extremely flat band in bilayer graphene, Sci. Adv. 4, 0059 (2018).

14 S. Carr, Shiang Fang, P. Jarillo-Herrero, and E. Kaxiras, Pressure dependence of the magic twist angle in graphene superlattices, Phys. Rev. B 98, 085144 (2018).

15 Yuan Cao, V. Fatemi, Shiang Fang, K. Watanabe, T. Taniguchi, E. Kaxiras and P. Jarillo-Herrero, Unconven- tional superconductivity in magic-angle graphene superlattices, Nature (2018), doi:10.1038/nature26160.

16 Yuan Cao, V. Fatemi, A. Demir, Shiang Fang, S.L. Tomarken, J.Y. Luo, J.D. Sanchez-Yamagishi, K. Watanabe, T. Taniguchi, E. Kaxiras, R.C. Ashoori and P. JarilloHerrero, Correlated insulator behaviour at half-filling in magic-angle graphene superlattices, Nature (2018), doi:10.1038/nature26154.

17 A. Legros, S. Benhabib, W. Tabis, F. Laliberte, M. Dion, M. Lizaire, B. Vignolle, D. Vignolles, H. Ray, Z. Z. Li, P. Auban-Senzier, N. Doiron-Leyraud, P. Fournier, D. Colson, L. Taillefer, and C. Proust, Universal T-linear resistivity and Planckian dissipation in overdoped cuprates, Nat. Phys. 15, 142 (2018),

18 Y. Nakajima, T. Metz, C. Eckberg, K. Kirshenbaum, A. Hughes, R. Wang, L. Wang, S. R. Saha, I.-L. Liu, N. P. Butch, D. Campbell, Y. S. Eo, D. Graf, Z. Liu, S. V. Borisenko, P. Y. Zavalij, and J. Paglione, Planckian dissipation and scale invariance in a quantum-critical disordered pnictide, arXiv:1902.01034

19 Y. Cao, D. Chowdhury, D. Rodan-Legrain, O. RubiesBigorda, K. Watanabe, T. Taniguchi, T. Senthil, and P. Jarillo-Herrero, Strange metal in magic-angle graphene with near Planckian dissipation, arXiv:1901.03710.

20 P. T. Brown, D. Mitra, E. Guardado-Sanchez, R. Nourafkan, A. Reymbaut, C.-D. Hebert, S. Bergeron, A. M. S. Tremblay, J. Kokalj, D. A. Huse, P. Schau and W. S. Bakr, Bad metallic transport in a cold atom FermiHubbard system, Science 363, 379 (2019).

21 V. R. Shaginyan, K. G. Popov, and V. A. Khodel, Quasiclassical physics and T-linear resistivity in both strongly correlated and ordinary metals, Phys. Rev. B 88, 115103 (2013).

22 V.A.Khodel, J.W.Clark, M.V.Zverev, Toward a topological scenario for high-temperature superconductivity of copper oxides, Phys. Lett. A 382, 3281-3286 (2018).

23 V.R. Shaginyan, M.Ya. Amusia, A.Z. Msezane, V.A. Stephanovich, G.S. Japaridze, and S.A. Artamonov, Fermion condensation, T-linear resistivity and Planckian limit, arXiv:1907.06921. 